\title{
48.
}

\section{NOTE SUR LES FONCTIONS DE M. STURM.}

[From the Journal de Mathématiques Pures et Appliquées (Liouville), tom. xI. (1846), pp. 297-299.]

ON sait que la suite des fonctions

$$
\begin{aligned}
& f x=(x-a)(x-b)(x-c)(x-d) \ldots, \\
& f_{1} x=\Sigma(x-b)(x-c)(x-d) \ldots, \\
& f_{2} x=\Sigma(a-b)^{2}(x-c)(x-d) \ldots, \\
& f_{3} x=\Sigma(a-b)^{2}(b-c)^{2}(c-a)^{2}(x-d) \ldots,
\end{aligned}
$$

est de la plus grande utilité dans la théorie de la résolution numérique des équations. En effet, on obtient tout de suite à leur moyen le nombre de racines réelles comprises entre deux limites quelconques. Il était donc intéressant de chercher la manière d'exprimer ces fonctions par les coefficients de $f x$.

Soit, pour cela, $m$ un nombre quelconque, pas plus grand que le degré $n$ de cette fonction. En prenant $k$ pour $m^{\text {ième }}$ racine de la suite $a, b, c, \ldots$, et mettant, pour abréger,

$$
P=(-)^{\frac{1}{2} m(m-1)}(a-b)(a-c) \ldots(a-k)(b-c) \ldots(b-k) \ldots(j-k),
$$

cela donne

$$
f_{m} x: f x=\mathbf{\Sigma} \frac{P^{2}}{(x-a)(x-b) \ldots(x-k)}
$$

dans laquelle expression

$$
P=\left|\begin{array}{ccc}
1, & a, \ldots, & a^{m-1} \\
1, & b, \ldots, & b^{m-1} \\
\ldots \ldots \ldots \ldots \ldots \ldots \ldots \\
1, & k, \ldots, & k^{m-1}
\end{array}\right|
$$


$48]$

NOTE SUR LES FONCTIONS DE M. STURM.

307

et, de plus,

$$
\frac{P}{(x-a)(x-b) \ldots(x-k)}=(-)^{\frac{1}{2} m(m-1)}\left[\frac{(b-c) \ldots(b-k) \ldots(j-k)}{(x-a)}+\ldots\right],
$$

dans laquelle le coefficient de $x^{-r}$ est égal à

$$
(-)^{\frac{1}{2} m(m-1)}\left[a^{r-1}(b-c) \ldots(b-k) \ldots(j-k)+\ldots\right],
$$

c'est-à-dire à

$$
\left|\begin{array}{cccc}
1, & a, \ldots, & a^{m-2}, & a^{r-1} \\
1, & b, \ldots, & b^{m-2}, & b^{r-1} \\
\ldots \ldots \ldots \ldots \ldots \ldots \ldots \ldots \ldots \ldots \\
1, & k, \ldots, & k^{m-2}, & k^{r-1}
\end{array}\right|
$$

Donc enfin le coefficient de $x^{-r}$ dans $f_{m} x: f x$ est égal à

$$
\boldsymbol{\Sigma}\left|\begin{array}{rrr}
1, & a, \ldots, & a^{m-1} \\
\ldots \ldots \ldots \ldots \ldots \ldots \ldots \\
1, & k, \ldots, & k^{m-1}
\end{array}\right| \times\left|\begin{array}{cccc}
1, & a, \ldots, & a^{m-2}, & a^{r-1} \\
\ldots \ldots \ldots \ldots \ldots \ldots \ldots \ldots \ldots \\
1, & k, \ldots, & k^{m-2}, & k^{r-1}
\end{array}\right|
$$

où, au moyen d'une propriété connue des déterminants, en représentant comme à l'ordinaire par $S_{q}$ la somme des $q^{\text {ièmes }}$ puissances de toutes les racines, ce coefficient devient égal à

$$
\left|\begin{array}{lrrr}
S_{0_{i}}, & S_{1}, \ldots, & S_{m-2}, & S_{r-1} \\
S_{1}, & S_{2}, \ldots, & S_{m-1}, & S_{r} \\
\ldots \ldots \ldots \ldots \ldots \ldots \ldots \ldots \ldots \ldots \ldots \ldots \ldots \ldots \ldots \ldots \ldots & \ldots \ldots \ldots, \\
S_{m-1}, & S_{m}, \ldots, & S_{2 m-3}, & S_{r+m-2}
\end{array}\right|
$$

De là, en mettant

$$
T_{q}=\Sigma \frac{a^{q}}{x-a}
$$

$$
f_{m} x: f x=\left|\begin{array}{lrrr}
S_{0}, & S_{1}, \ldots, & S_{m-2}, & T_{0} \\
S_{1}, & S_{2}, \ldots, & S_{m-1}, & T_{1} \\
\ldots \ldots \ldots \ldots \ldots \ldots \ldots \ldots \ldots \ldots \ldots \ldots \ldots \ldots \ldots & \ldots \ldots \ldots \ldots \\
S_{m-1}, & S_{m}, \ldots, & S_{2 m-3}, & T_{m-1}
\end{array}\right|
$$

en multipliant par $f x$, et mettant

$$
Q_{m, r}=S_{m+r-1}-p_{1} S_{m+r=2} \ldots+(-)^{r} p_{r} S_{m-1},
$$

où l'on suppose

$$
f x=x^{n}-p_{1} x^{n-1} \ldots+(-)^{n} p_{n},
$$

on obtient

$$
f_{m} x=\sum_{r} x^{n-m-r}\left|\begin{array}{llll}
S_{0}, & S_{1}, \ldots, & S_{m-2}, & Q_{m, r} \\
S_{1}, & S_{2}, \ldots, & S_{m-1}, & Q_{m+1, r} \\
\ldots \ldots \ldots \ldots \ldots \ldots \ldots \ldots \ldots \ldots \ldots \ldots \ldots \ldots \ldots \ldots \ldots \ldots \ldots \ldots \ldots \ldots & \ldots \ldots \ldots \\
S_{m-1}, & S_{m}, \ldots, & S_{2 m-3}, & Q_{2 m-1, r}
\end{array}\right|
$$

$39-2$

www.rcin.org.pl 
où $r$ peut ne s'étendre que depuis 0 jusqu'à $n-m$, puisque $f_{m} x$ est fonction entière. Au moyen des relations connues qui existent entre les quantités $S_{q}$, on a

$$
\begin{aligned}
Q_{m+8, r} & =(-)^{r} p_{r+1} S_{m+8-2} \ldots+(-)^{n-1} p_{n} S_{r+m+8-n-1} \ldots \quad[r+m+s>n] \\
Q_{m+8, r} & =(-)^{r} p_{r+1} S_{m+8-2} \ldots+(-)^{r+m+8-3} p_{r+m+8-2} S_{1} \\
& +(-)^{r+m+8-2} p_{r+m+8-1}(r+m+s-1) \ldots \ldots \ldots \ldots\left[r+m+s_{<}=n\right]
\end{aligned}
$$

et de là, en posant

$$
\begin{aligned}
Q_{m+s, r}^{\prime} & =(-)^{r+m-1} p_{r+m} S_{8-1} \ldots+(-)^{n-1} p_{n} S_{r+m+8-n-1} \ldots[r+m+s>n], \\
Q_{m+8, r}^{\prime} & =(-)^{r+m-1} p_{r+m} S_{s-1} \ldots+(-)^{r+m+s-3} p_{r+m+8-2} S_{1} \\
& +(-)^{r+m+8-2} p_{r+m+8-1}(r+m+s-n-1) \ldots\left[r+m+s_{<}^{n}\right],
\end{aligned}
$$

on peut, par les propriétés des déterminants, réduire $Q_{m+s, r}$ à $Q_{m+s, r}^{\prime}$ dans l'expression de $f_{m} x$. Nous avons done exprimé cette fonction au moyen des coefficients $p_{1}, p_{2}$, et des sommes $S_{1}, S_{2}, \ldots, S_{2 m-3}$, lesquelles s'expriment facilement par ces mêmes coefficients (et pour calculer $f_{1} x, f_{2} x, \ldots, f_{n} x$, on aurait seulement besoin de calculer ces sommes une fois pour toutes jusqu'à $S_{2 n-3}$ ); il serait donc facile de former des tables de ces fonctions, pour les équations d'un degré quelconque, ce qui pourrait à peine s'effectuer d'aucune autre manière. 